\title{
Commentaire
}

\section{Le démon de Laplace est mort, vive les génies de la place !}

\author{
Cyrille Rigolot \\ Zootechnicien, INRA, UMR Métafort, 63122 Saint-Genès Champanelle, France
}

Dans l'article de Guillaume Deffuant et al. ${ }^{1}$, les auteurs, associés au réseau national des Systèmes complexes (RNSC) et à la Complex Systems Society ${ }^{2}$, nous livrent trois visions de la complexité. Ils s'appuient pour ceci sur l'image du démon de Laplace, capable de prédire l'avenir avec exactitude, à partir d'une connaissance exhaustive de l'état d'un système. La vision 1 résiste au démon (mécanique quantique, théorie du chaos...) ; la vision 2 reconnaît ces limites, mais continue d'utiliser le principe de démon pour des systèmes limités, en mobilisant la masse de données aujourd'hui disponible et les nouvelles possibilités technologiques de traitement; la vision 3 « met le démon face au défi de simuler la subjectivité et ses effets collectifs ». Destiné au lectorat interdisciplinaire de NSS, le point de vue des auteurs a des implications sur la façon d'envisager l'interdisciplinarité au-delà de la communauté des modélisateurs informatiques, et en collaboration avec eux. Pour approfondir cet effort de clarification, je voudrais ici apporter le point de vue d'un chercheur sur les systèmes agricoles, mobilisant parfois la modélisation informatique. De ce point de vue particulier, la vision 2 proposée par Deffuant $e t$ al. présente un intérêt heuristique notable, alors que la vision 3 porte une ambigüité.

Les systèmes agricoles sont des systèmes complexes, marqués par les aléas et les interactions, particulièrement entre les domaines social et biologique. De façon cohérente avec la vision 2, une partie des recherches dans le domaine agronomique est marquée par le développement de puissants outils de simulation intégrés. Un des enjeux est de développer des approches multicritères et une représentation toujours plus large et plus fine, de façon à avoir la vision la plus exhaustive possible des systèmes. Dans cette optique, par exemple, la plateforme Record (Bergez et al., 2013) fondée sur le formalisme DEVS (discrete event system specification) (Zeigler, 1987) est une innovation permettant de faire le lien entre des niveaux d'organisation allant des organismes végétaux et animaux aux territoires, sous réserve de disponibilité de données pour le paramétrage des simulations. $\mathrm{Au}$ niveau international, une illustration est la nouvelle génération d'évaluations intégrées (global integrated assessment) qui rassemble dans des simulateurs sophistiqués des modèles climatiques, macroéconomiques, biologiques et des bases de données détaillées des systèmes agricoles (Herrero et al., 2015). L'image du démon de Laplace présente un intérêt heuristique pour décrire ce courant de recherche et faciliter la mise en place de cette forme d'interdisciplinarité autour de l'outil de simulation.

Deffuant $e t$ al. font la distinction entre leurs visions 2 et 3 de la complexité sur la base de la subjectivité des acteurs. Sans qu'elle soit définie précisément, cette subjectivité est associée dans leur propos à la décision. En agronomie, la décision des acteurs a longtemps été une ligne de partage entre deux visions de la complexité. De façon bien illustrative, la décision a longtemps été en France un objet de recherche spécifique du département Sciences pour l'action et le développement (SAD) de l'Inra (Sebillote et Soler, 1990), identifié au sein de l'institut par une vision bien particulière de l'interdisciplinarité (Deffontaines et Hubert, 2004). Cependant, alors que la possibilité ou non de prédire la subjectivité est présentée par G. Deffuant et al. comme un débat interne au sein de la communauté des sciences humaines et sociales, elle a de mon point de vue des conséquences fondamentales

\footnotetext{
Auteur correspondant : cyrille.rigolot@clermont.inra.fr

1 GuillaumeDeffuant et al. «Visions de la complexité. Le démon de Laplace dans tous ses états » (NSS, 23, 1, 42-53).

2 http://cssociety.org.
} 
sur le champ entier de la connaissance. En fait, les développements informatiques permettent $\mathrm{d}$ 'ores et déjà de simuler la décision, en tenant compte des préférences des acteurs ou de l'aversion au risque. Un exemple est le modèle Mélodie à l'échelle de l'exploitation de polyculture-élevage (Chardon et al., 2012), fondé sur une ontologie des systèmes pilotés et la plateforme Diese (MartinClouaire et Rellier, 2009). Dès lors que la simulation de la décision est possible, rien n'empêche de l'intégrer dans des modèles toujours plus complexes. Ainsi, le modèle Mélodie a été transféré sur la plateforme Record (Bergez et al., 2013). De même, à l'échelle internationale, M.T. van Wijk (2014) propose d'inclure la décision des agriculteurs et d'autres acteurs des petits territoires dans les évaluations intégrées (global integrated assessment) en utilisant des summary functions dérivées de modèles multiagents ou d'autres méthodologies. Ainsi, la prise en compte de la décision n'est pas en soi incompatible avec la vision 2 de Deffuant et al. qui continue de mobiliser le principe du démon à des échelles restreintes.

De mon point de vue, une différence fondamentale tient non pas à l'objet de la modélisation informatique mais à ses objectifs et à son insertion dans un processus de recherche-développement. Pour clarifier ce point, je voudrais expliciter une vision de la complexité où le démon de Laplace est mort. Dans cette vision, la modélisation informatique reste utile, cependant son objectif n'est plus la prédiction mais les apprentissages. Cette vision fait le lien entre «complexité restreinte » (l'objet de la communauté scientifique des auteurs) et la " complexité généralisée » au sens d'Edgar Morin (2007), comme le propose P. Roggero (2008). La complexité généralisée considère que tout système est complexe dans sa nature même, et la subjectivité y occupe une place fondamentale (Morin, 1980). Dans cette optique, une reformulation de Deffuant $\mathrm{et} \mathrm{al}$. pourrait être la suivante: la vision 1 non pas «résiste » au démon de Laplace, mais plus radicalement ruine son principe dans ses fondements (Morin, 2007). La vision 2 est une approche qui en elle-même n'a pas fait son deuil du démon. La vision 3 constitue une ouverture sur la complexité généralisée, mais elle tend à la refermer aussitôt, en mettant le démon au défi de simuler la subjectivité pour la prédire. En reconnaissant très explicitement le caractère fondamentalement non prédictible des systèmes complexes, la modélisation informatique reste un outil précieux pour apprendre et accompagner les transformations (Étienne, 2010 [cité par les auteurs] ; Rigolot et al., 2015). Dans cette perspective, les modèles informatiques pertinents peuvent être simples ou très sophistiqués, intégrer la décision ou pas, le plus important étant la façon dont ils sont mobilisés. Celle-ci doit être participative et contextualisée, pour faire émerger les forces créatrices subjectives des acteurs dans leurs territoires : les génies de la place.

\section{Bibliographie}

Bergez, J.-E., Chabrier, P., Gary, C., Jeuffroy, M.-H., Makowski, D., Quesnel, G., Ramat, E., Raynal, H., Rousse, N., Wallach, D., Debaeke, P., Durand, P., Duru, M., Dury, J., Faverdin, P., C. Gascuel-Odoux, C., Garcia, F., 2013. An open platform to build, evaluate and simulate integrated models of farming and agro-ecosystems, Environmental Modelling $\mathcal{E}$ Software, 39, 39-49.

Chardon, X., Rigolot, C., Baratte, C., Espagnol, S., Raison, C., Martin-Clouaire, R., Rellier, J.-P., Le Gall, A., Dourmad, J.-Y., Piquemal, B., Leterme, P., Paillat, J.-M., Delaby, L., Garcia, F., Peyraud, J.-L., Poupa, J.-C., Morvan, T., Faverdin, P., 2012. MELODIE: a whole-farm model to study the dynamics of nutrients in dairy and pig farms with crops, Animal, 6, 10, 1711-1721.

Deffontaines, J.-P., Hubert, B., 2004. Dossier Interdisciplinarité. Un regard sur l'interdisciplinarité à l'Inra. Point de vue de deux chercheurs du département Sciences pour l'action et le développement (SAD). Natures Sciences Sociétés, 12, 2, 186-190.

Étienne, M. (Ed.), 2010. La modélisation d'accompagnement. Une démarche participative en appui au développement durable, Versailles, éditions Quæ.

Herrero, M., Wirsenius, S., Henderson, B., Rigolot, C., Thornton, P., Havlik, P., de Boer, I., Gerber, P., 2015. Livestock and the Environment: What Have We Learned in the Last Decade?, Annual Review of Environment and Resources, 40, 1, 177-202.

Martin-Clouaire, R., Rellier, J.-P., 2009. Modelling and simulating work practices in agriculture. International Journal of Metadata, Semantics and Ontologies, 4, 1-2, 42-53.

Morin, E., 1980. La méthode. Tome 2. La Vie de la Vie. Paris, Seuil.

Morin, E., 2007. Complexité restreinte, complexité générale. In Le Moigne, J.-L., Morin, E., (Eds), Intelligence de la complexité. Épistémologie et pragmatique, Paris, Éditions de l'Aube, 2007, 28-64.

Rigolot, C., Watson, I., Herrero, M., Delma, B.J., Vall, E., Andrieu, N., Yakouba, B., Ouédraogo, S., Ziebe, R., Dowe, V., Kolyang, T., Prestwidge, D., McDonald, C., Stirzaker, R., Bruce, C., Carberry, P., 2015. Modelling households and value chains: Complementary methods for learning and evaluation in innovations platforms. Conférence internationale sur les systèmes d'innovation en Afrique de l'Ouest et du Centre, 25-27 Février 2015, Saly Portudal (Sénégal).

Roggero, P., 2008. Pour une sociologie d'après "La Méthode ", Communications, 82, 1, 143-159.

Sebillotte, M., Soler, L.-G., 1990. Les processus de décision des agriculteurs. In Brossier, J., Vissac, B., Le Moigne J.-L., (Eds), Modélisation systémique et système agraire. Décision et organisation, Actes du Séminaire du département de recherches sur les Systèmes agraires et le développement (SAD), SaintMaximin, 2-3 mars 1989, Versailles, Inra-Sad, 93-101, http://infodoc.agroparistech.fr/index.php?lvl=author_ see\&id $=90103$.

Wijk, M. T. (van), 2014. From global economic modelling to household level analyses of food security and sustainability: How big is the gap and can we bridge it?, Food Policy, 49, 2, 378-388.

Zeigler, B., 1987. Hierarchical, modular discrete-event modelling in an object-oriented environment, Simulation, 49, 5, 219-230. 